\title{
Shortening Postoperative Stay after Parathyroidectomy - A District General Hospital Experience
}

\author{
Sidhartha Sinha ${ }^{1,2}$ Matthew Fok ${ }^{1}$ ljaz Ahmad ${ }^{2}$ Mustafa Al-Sheikh ${ }^{2}$ Christopher Backhouse ${ }^{2}$ \\ ${ }^{1}$ Department of General and Vascular Surgery, Basildon and Thurrock \\ University Hospitals NHS Foundation Trust, Basildon, United \\ Kingdom of Great Britain and Northern Ireland \\ 2 Department of General and Vascular Surgery, Colchester Hospital \\ University NHS Foundation Trust, Colchester, Essex, United Kingdom \\ Address for correspondence Sidhartha Sinha, FRCS, Department of \\ General and Vascular Surgery, Basildon and Thurrock University \\ Hospitals NHS Foundation Trust, Nethermayne, Basildon \\ (e-mail: sidhartha.sinha@nhs.net).
} of Great Britain and Northern Ireland

Int Arch Otorhinolaryngol 2020;24(3):e313-e318.

Abstract


Keywords
- Parathyroidectomy
- length of stay
- surgical outcomes
- quality improvement
- endocrine
- head and neck
surgery
- short stay surgery

Introduction Historically, concerns about complications following parathyroid surgery, such as airway compromise, bleeding and hypocalcemia, have precluded its consideration as a short-stay surgical procedure. Recent advancements in perioperative care have resulted in several publications demonstrating that parathyroidectomy can be safely performed as a short-stay procedure.

Objectives The aim of the present study was to describe the process of implementing a short-stay protocol focusing on preoperative patient education and postoperative calcium management for those undergoing surgery for primary hyperparathyroidism (PHP).

Method A retrospective audit of consecutive parathyroidectomies performed for PHP over the period between 2010 and 2013 was performed. A short-stay protocol (SSP) was introduced focusing on postoperative calcium management. Results were reaudited over the period between 2013 and 2015.

Results Consecutive parathyroidectomies in 76 patients were included in the study. A total of 42 patients underwent parathyroidectomy prior to the introduction of the protocol. A total of $26.2 \%$ of these patients were symptomatic from hypercalcemia. A total of 40 out of $42(95.2 \%)$ patients had a biochemical cure. A total of 36 out of $42(85.7 \%)$ cases were due to parathyroid adenomas. A total of 34 patients underwent surgery following the introduction of the protocol. A total of 13 out of $34(38.2 \%)$ of the patients had symptomatic hypercalcemia. A total of 33 out of $34(97.1 \%)$ had a biochemical cure. A total of 32 out of 34 (94.1\%) cases were due to parathyroid adenomas.

The length of stay decreased from a median of 3 days (range 2-9 days; mean 3.32) preprotocol to a median of 2 days (range 2-3 days; mean 2.16) postprotocol $(p<0.0001)$ with no difference in the 30 -day unplanned readmission rate (4.8 versus $2.9 \% ; p=0.999)$.

Conclusions The postoperative length of stay after parathyroidectomy for PHP can be safely reduced through patient education and by rationalizing postoperative calcium management without adversely affecting outcomes. received

January 20, 2019

accepted

September 8, 2019

published online

December 13, 2019
DOI https://doi.org/

10.1055/s-0039-1698777. ISSN 1809-9777.
Copyright $\odot 2020$ by Thieme Revinter

Publicações Ltda, Rio de Janeiro, Brazil
License terms

()ㅇㅇ $\Theta \circledast$ 


\section{Introduction}

Primary hyperparathyroidism (PHP) is a common endocrine disorder with incidence rates of between 1 and $2 \%$ in certain subsets of the population. ${ }^{1}$ Parathyroidectomy remains the only method to cure PHP. However, in the UK, parathyroidectomy has historically been excluded from day-case or short-stay surgery due a reliance on Kocher-type full-cervical incisions and consequent concerns about airway-compromise and postoperative bleeding, as well as symptomatic postoperative hypocalcemia. ${ }^{2,3}$ Despite these concerns, several studies from high-volume, academic centers have demonstrated that parathyroidectomy can be safely performed with discharge on the day of surgery or on the $1^{\text {st }}$ postoperative day. ${ }^{3-10}$ The purpose of the present study was to report the results of parathyroidectomy at a 'mediumvolume' endocrine unit in a District General Hospital setting before and after the implementation of a protocol intended to reduce the postoperative length of stay (LOS) by focusing on periprocedural patient education and postoperative management of calcium monitoring and supplementation.

\section{Methods}

\section{Patient Cohorts}

This was a single-center audit and service reconfiguration project comprising a retrospective review of historical outcomes preceding service change, the introduction of a new patient-pathway and a prospective reaudit of outcomes. The historical cohort comprised consecutive patients undergoing parathyroidectomy for PHP from 2010 to 2013. Redo cases and cases with concomitant thyroid pathology requiring surgery were excluded from the cohort. The following cohort comprised consecutive patients with PHP managed under the new short-stay protocol (SSP). All of the surgeries were performed by a single general surgeon with a vascular and endocrine subspecialty interest.

\section{Procedural Technique}

The following steps remained constant throughout the study period. All of the patients underwent dual modality preoperative imaging with sestamibi-technetium 99m scintigraphy (MIBI) and cervical ultrasonography (USS). Computed tomography (CT) was utilized selectively (that is, in cases of suspected mediastinal disease). Patients with concordant USS and MIBI imaging that demonstrated single gland disease underwent targeted (hemicervical) incision. Those with concordant imaging demonstrating multigland disease (or where both modalities failed to identify parathyroid pathology) underwent traditional full-length Kocher cervical incision to allow bilateral neck exploration (BNE) if needed. Those with discordant imaging underwent either targeted or traditional full-length incision. Laryngoscopy was not routinely used preoperatively and was only used postoperatively if recurrent laryngeal nerve injury was suspected clinically. All of the cases were performed under general anesthetic with endotracheal intubation. Frozen section and wound drains were used selectively at the discretion of the operating surgeon.

\section{Perioperative Care Prior to the Introduction of the Short-stay Protocol}

All of the patients were admitted to a surgical ward postoperatively. Postoperative serum calcium monitoring was performed at 6 hours, 12 hours, 24 hours, 48 hours, on the $5^{\text {th }}$ post-operative day (the latter as an outpatient), and at 6 weeks. Skin closure was performed with a nonabsorbable suture (Prolene, Ethicon Inc., Somerville, NJ, USA) which was removed at the bedside on the morning of the $3^{\text {rd }}$ postoperative day. Patients were discharged with instructions to return to the outpatient phlebotomy department on the $5^{\text {th }}$ postoperative day for a serum calcium check. Patients were not routinely discharged with oral calcium supplementation.

\section{Short-stay Protocol}

Central to the SSP was the rationalization of postoperative serum calcium monitoring, modification of other factors contributing to inpatient stay (that is, skin closure technique and medical/nursing team education) and preoperative patient education to expect expedited discharge. The SSP was created in collaboration with the senior nursing staff from the General Surgical directorate.

Skin closure was changed from nonabsorbable to absorbable (Monocryl, Ethicon Inc., Somerville, NJ, USA) suture material while inpatient calcium monitoring was reduced to checks at 6 hours and 24 hours and outpatient phlebotomy attendance for 48 hour, day 5 and 6 week checks. Long term follow-up was maintained as for the pre-SSP cohort. All of the patients were discharged with oral calcium supplements with individualized plans for administration depending on their postoperative calcium results and generic instructions regarding further supplementation in the event of unexpected hypocalcemic symptoms. The patients were counseled at their initial outpatient clinic consultation and preadmission assessment about the SSP and were provided with a custom-written patient information leaflet with contact numbers for out-of-hours support (-Appendix A). The general surgical directorate was engaged through lecture presentations prior to the institution of the SSP and further supported with custom-written handover sheets (-Appendix B). All of the new patients with PHP were considered for the SSP, with exclusion criteria shown in - Table 1.

\section{Statistical Analysis}

Continuous variables are given as median (with range) and mean for information. The univariate comparison between groups was performed using the Mann-Whitney U-test. Categorical variables are presented as percentages with comparisons performed using the Fisher exact test. Data was analyzed using IBM SPSS Statistics for Windows, Version 20.0 (IBM Corp., Armonk, NY, USA). Statistical significance was set as $p<0.05$. 
Table 1 Exclusion criteria for short-stay protocol

\begin{tabular}{|l|}
\hline Exclusion criteria for short-stay protocol \\
\hline Current in-patients (LOS* inappropriate metric) \\
\hline ASA* grade III or above $^{*}$ Subjectively infirm/immobile \\
\hline $\begin{array}{l}\text { Subjectively inadequate social support (e.g., living } \\
\text { alone, no contact telephone number) }\end{array}$ \\
\hline $\begin{array}{l}\text { Suspected MEN* syndrome, secondary or tertiary } \\
\text { hyperparathyroidism }\end{array}$ \\
\hline $\begin{array}{l}\text { Combined procedure (e.g., parathyroidectomy and thyroid } \\
\text { lobectomy) }\end{array}$ \\
\hline $\begin{array}{l}\text { Extended procedure (e.g., mediastinal exploration or total } \\
\text { parathyroidectomy) }\end{array}$ \\
\hline Redo surgery \\
\hline
\end{tabular}

Abbreviations: ASA, American Society of Anesthesiologists; LOS, length of stay; MEN, multiple endocrine neoplasia.

\section{Results}

A total of 76 patients were included in the study (42 pre-SSP and 34 post-SSP). Baseline demographics are listed in - Table 2 and indicated no significant differences between the groups. Both patient cohorts were predominantly female (76.2\% pre-SSP versus 76.5\% post-SSP, $p=0.999$ ) and asymptomatic (73.8\% pre-SSP versus $61.8 \%$ post-SSP, $p=0.324$ ) with comparable preoperative serum calcium and parathyroid hormone levels. The USS was positive in 36 out of 42 (85.7\%) pre-SSP patients and in 29 out of 34 (85.3\%) post-SSP patients. The MIBI scan was positive in 38 out of 42 (90.5\%) pre-SSP patients and in 30 out of 33 (90.9\%) post-SSP patients. Concordance was similar in the 2 cohorts $(73.8 \%$ pre-SSP versus $85.3 \%$ post-SSP).

The postoperative results are listed in - Table 3. A targeted incision was used in 30 out of 42 (71.4\%) patients of the preSSP group and in 27 out of 34 (79.4\%) patients of the post-SSP group $(p=0.595)$. There were four cases of conversion to fulllength cervical incision (BNE), all occurring in the pre-SSP

Table 2 Baseline demographics for pre-SSP and post-SSP patient cohorts

\begin{tabular}{|l|l|l|l|}
\hline Variable & Pre-SSP & Post-SSP & -value \\
\hline Number & 42 & 34 & N/A \\
\hline Female (n, \%) & $32(76.2 \%)$ & $26(76.5 \%)$ & 0.999 \\
\hline Median (range) / mean age (years old) & $69(41-92) / 67.5$ & $65.5(20-85) / 62$ & 0.122 \\
\hline ASA I or II (n, \%) & $39(92.9 \%)$ & $34(100 \%)$ & 0.248 \\
\hline Asymptomatic (n, \%) & $31(73.8 \%)$ & $21(61.8 \%)$ & 0.324 \\
\hline Median (range) / mean preoperative PTH (pmol/L) & $14.3(5.2-127.1) / 23.9$ & $14.9(6.9-44.2) / 16.6$ & 0.859 \\
\hline Median (range) / mean preoperative Ca ${ }^{2+}$ (mmol/L) & $2.89(2.68-3.49) / 2.93$ & $2.82(2.63-3.37) / 2.85$ & 0.060 \\
\hline Concordant imaging (n, \%) & $31(73.8 \%)$ & $29(85.3 \%)$ & 0.267 \\
\hline
\end{tabular}

Abbreviations: ASA, American Society of Anesthesiologists; $\mathrm{Ca}^{2+}$, corrected serum calcium; PTH, parathyroid hormone; SSP, short-stay protocol.

Table 3 Outcomes for pre-SSP and post-SSP patient cohorts

\begin{tabular}{|l|l|l|l|}
\hline Variable & Pre-SSP $(\boldsymbol{n}=42)$ & Post-SSP $(\boldsymbol{n}=34)$ & $p$-value \\
\hline Targeted incision (n, \%) & $30(71.4 \%)$ & $27(79.4 \%)$ & 0.595 \\
\hline $\begin{array}{l}\text { Median (range) / mean number of glands removed } \\
\text { or biopsied }\end{array}$ & $1(1-4) / 1.81$ & $1(1-4) / 1.18$ & $<0.0001$ \\
\hline Histopathology = adenoma (n, \%) & $36(85.7 \%)$ & $32(94.1 \%)$ & 0.285 \\
\hline Median (range) / mean day 5 Ca ${ }^{2+}(\mathrm{mmol} / \mathrm{L})$ & $2.42(1.55-2.87) / 2.38$ & $2.35(2.07-2.54) / 2.34$ & 0.017 \\
\hline Median (range) / mean 6 week Ca ${ }^{2+}(\mathrm{mmol} / \mathrm{L})$ & $2.44(2.20-2.81) / 2.44$ & $2.41(1.85-2.84) / 2.42$ & 0.889 \\
\hline Biochemical cure** $(\mathrm{n}, \%)$ & $40(95.2 \%)$ & $33(97.1 \%)$ & 0.999 \\
\hline Post-operative temporary hypocalcaemia*** $(\mathrm{n}, \%)$ & $3(7.1 \%)$ & $2(5.9 \%)$ & 0.999 \\
\hline Other complications ${ }^{* * * *}(\mathrm{n}, \%)$ & $2(4.8 \%)$ & $2(2-3) / 2.16$ & 0.399 \\
\hline Median (range) / mean LOS**** (days) & $3(2-9) / 3.32$ & $1(2.9 \%)$ & $<0.0001$ \\
\hline 30-day unplanned readmission ${ }^{* * * * * *}(\mathrm{n}, \%)$ & $2(4.8 \%)$ & & 0.999 \\
\hline
\end{tabular}

Abbreviations: $\mathrm{Ca}^{2+}$, corrected serum calcium; LOS, length of stay; SSP, short-stay protocol.

** includes patients with temporary hypocalcemia.

*** according to postoperative day 5 calcium result.

**** 2 cases of acute kidney injury, 2 cases of acute urinary retention, 1 cardiac arrhythmia, and 1 wound hematoma (managed conservatively).

***** length of stay (from admission to discharge).

****** all readmissions were due to symptomatic hypocalcemia. 

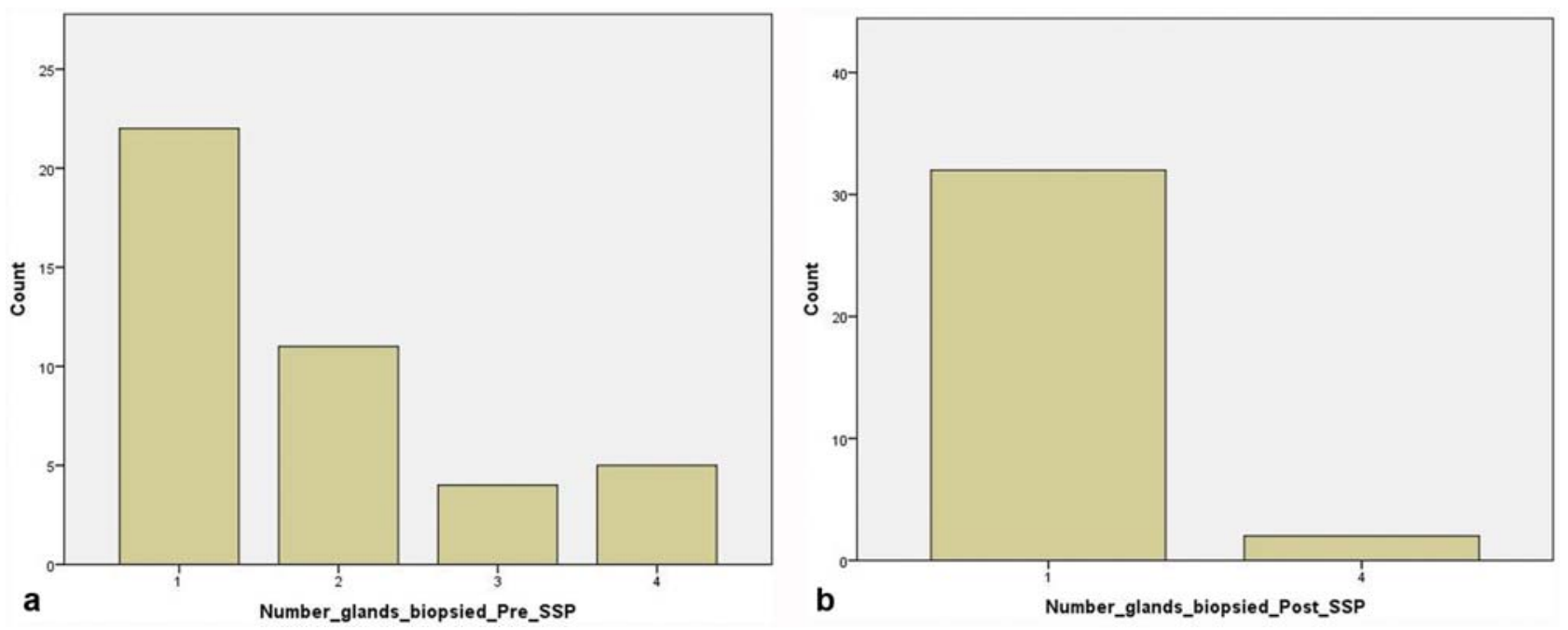

Fig. 1 (a) Number of glands biopsied per case pre-SSP. (b) Number of glands biopsied per case post-SSP.

cohort. A biochemical cure was achieved in 73 out of 76 (96.1\%) patients. A total of 2 out of $42(4.8 \%)$ patients in the pre-SSP group and of 1 out of 34 (2.9\%) patients in the postSSP group were persistently hypercalcemic at 6 weeks. Although the median number of glands removed per patient in both cohorts was 1 , there was a tendency to biopsy more glands per patient in the pre-SSP cohort compared with the post-SSP cohort (mean number 1.81 pre-SSP versus 1.18 post-SSP, $p=0.017$; $\mathbf{- F i g}$. $\mathbf{1}$ a and $\mathbf{1 b}$ ). The median postoperative day 5 corrected serum calcium levels were lower in the post-SSP group (2.42 mmol/L pre-SSP versus $2.35 \mathrm{mmol} / \mathrm{L}$ post-SSP, $p=0.017$ ) but this difference was not present at 6 weeks ( $2.44 \mathrm{mmol} / \mathrm{L}$ pre-SSP versus $2.41 \mathrm{mmol} / \mathrm{L}$ post-SSP, $p=0.889$ ). Parathyroid adenoma was the most common pathology found, and the proportions were similar in both groups ( $85.7 \%$ pre-SSP versus $94.1 \%$ post-SSP, $p=0.285$ ).

The median LOS decreased by $50 \%$ from 3 days to 2 days following the introduction of the protocol $(p<0.0001)$, as shown in - Fig. 2. In addition, the post-SSP cohort displayed

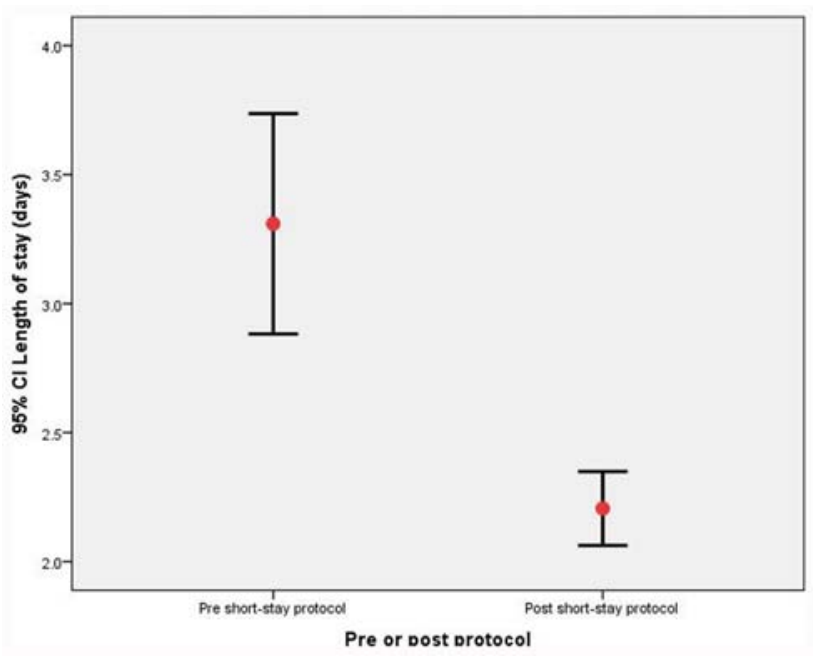

Fig. 2 Length of stay (days) pre- and post-SSP. much greater precision in LOS outcomes than the pre-SSP cohort, with no patient staying $>3$ days (-Fig. $\mathbf{3 a}$ and $\mathbf{3 b}$ ).

There were no differences in postoperative complication rates between the two cohorts. A total of 3 out of 42 (7.1\%) patients in the pre-SSP group were rendered temporarily hypocalcemic (at postoperative day 5) compared with 2 out of $34(5.9 \%)$ patients in the post-SSP group $(p=0.999)$. For the post-SSP group, 1 of the 2 patients was contacted and instructed to commence oral calcium supplementation based on their 48-hour calcium results (despite being asymptomatic), while the other complained of facial symptoms on day 5 and, therefore, was instructed to commence supplementation then. This latter patient (who turned out to have four gland parathyroid hyperplasias) continued to complain of symptoms despite oral supplementation and was readmitted on postoperative day 7 for intravenous calcium replacement. All but one of the five patients rendered temporarily hypocalcemic had undergone multigland biopsies during their surgery. One patient from each group remained hypocalcemic at 6 weeks. There were 2 unplanned readmissions within 30 days for the pre-SSP group compared with 1 (the aforementioned) readmission in the post-SSP group ( $4.8 \%$ versus $2.9 \%, p=0.999$ ). All three cases were due to symptomatic hypocalcemia. Other recorded complications were two cases of transient postoperative acute kidney injury in the pre-SSP group and two cases of acute urinary retention, one cardiac arrhythmia, and one wound hematoma (which was managed conservatively) in the post-SSP group. There were no cases of suspected or confirmed recurrent laryngeal nerve palsy.

\section{Discussion}

The results of the present study demonstrate that the LOS can be safely shortened after parathyroidectomy by influencing patient expectations through preoperative education and by rationalizing the management of postoperative calcium monitoring and supplementation. Historically, the surgical management of PHT included a postoperative LOS of 3 to 

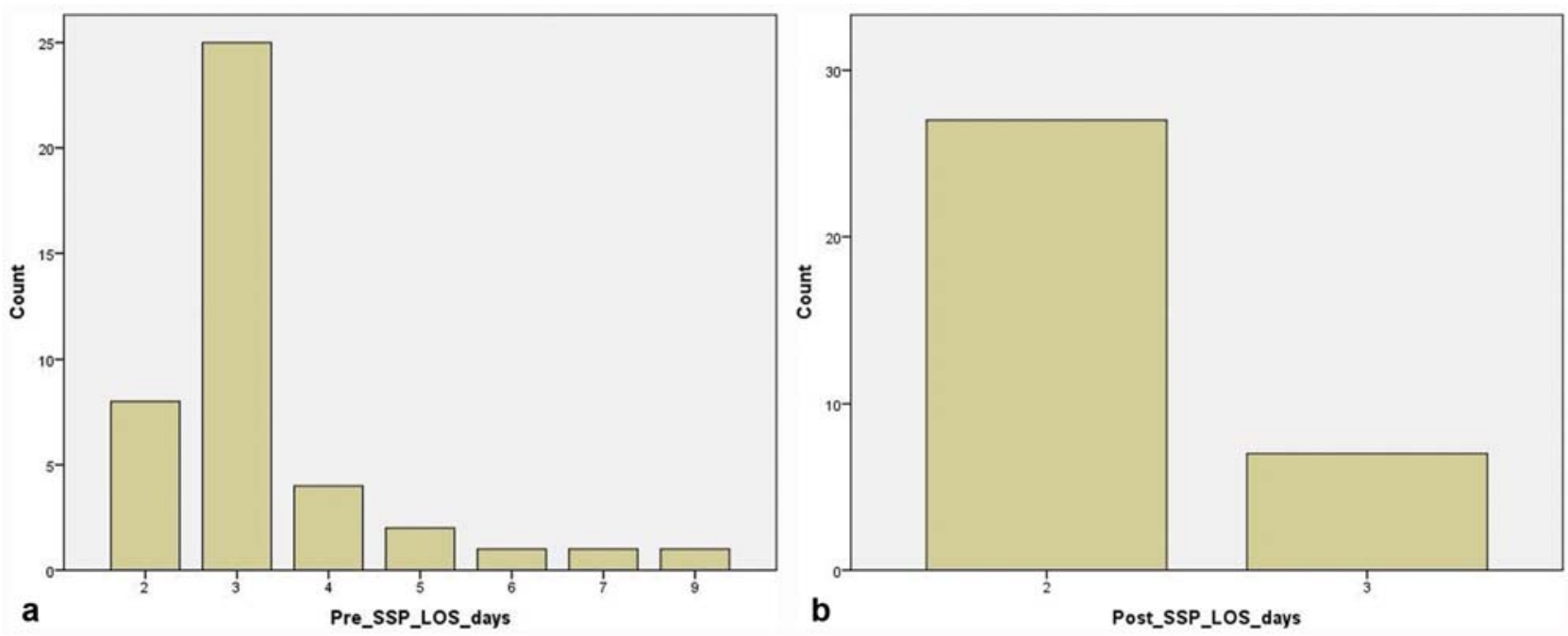

Fig. 3 (a) Range of lengths of stay (days) pre-SSP. (b) Range of lengths of stay (days) post-SSP.

4 days; however, recent national audits from the British Association of Endocrine and Thyroid Surgeons (BAETS) reported in 2012 that almost $80 \%$ of parathyroidectomy patients had a hospital stay of $<2$ days, while by 2017 , national data suggested that between 65 and $70 \%$ of the patients were being discharged within 24 hours of the surgery. ${ }^{11,12}$ Several studies have demonstrated that same-day discharge after parathyroidectomy is feasible with low rates of postoperative complications. ${ }^{3-8,10,13-16}$ Importantly, these reports acknowledge that accelerated discharge pathways are not appropriate for all patients undergoing parathyroidectomy.

Modalities that have been utilized to reduce postoperative stay after parathyroidectomy include focused or targeted incisions whereby preoperative imaging (most usually, a combination of MIBI and USS). ${ }^{17,18}$ Targeted exploration with incisions measuring between 2 and $3 \mathrm{~cm}$ that can be sited laterally rather than 'full-length' central incisions measuring between 5 and $6 \mathrm{~cm}$ have obvious cosmetic benefits and can be performed under local anesthetic, but it is postulated that the true advantage of these approaches are that they leave the remaining parathyroid glands undisturbed, reducing the risk of postoperative hypocalcemia secondary to iatrogenic parathyroid gland injury, and with the possibility of being performed without general anesthesia. ${ }^{3,18-20}$ Gland localization with perioperative sestamibi administration with the use of hand-held gamma probes and monitoring of intraoperative parathyroid hormone levels (ioPTH) is increasingly being used to confirm successful gland excision during targeted approaches; however, its cost-benefit is widely debated. ${ }^{4-6,8}$ Although high-grade evidence exists to support the benefit of both ultrasonic devices (Harmonic Scalpel, Ethicon Inc., Somerville, NJ, USA) and electrothermal bipolar devices (Ligasure, Medtronic, Dublin, Ireland) over the conventional "clamp-andtie" method in thyroid surgery, the use of advanced hemostatic devices to aid dissection and hemostasis in parathyroid surgery is more controversial. ${ }^{21,22}$

Concern about postoperative hypocalcemia and symptoms such as tetany after parathyroidectomy has tradition- ally been the reason why patients were managed as inpatients. ${ }^{5}$ The pathophysiology of postoperative hypocalcaemia is likely to be multifactorial. Damage to nondiseased parathyroid glands, suppression of function in normal glands from chronic high circulating levels of PTH and calcium and 'hungry-bone syndrome' (HBS, which describes a state of high calcium flux into skeletal tissue after PTH-driven osteoclastic resorption is abruptly terminated) have all been implicated. ${ }^{19,23-26}$ Despite knowledge of this condition, it is noteworthy that there remains discordance in practice from different units with regards to postoperative calcium supplementation, with some advocating routine and highly protocolized use, some reporting ad hoc usage dependent on surgeon preference, and others reporting no routine supplementation. $^{3-5,8,13,24}$

It is well-established that the use of clinical pathways can eliminate variations in care by encouraging multidisciplinary collaboration and through transparent signposting of expected processes of care and outcomes in the form of pathway documentation. ${ }^{27,28}$ With particular regard to parathyroidectomy, a clinical pathway incorporating clear patient selection criteria, preoperative patient education (in narrative written format and verbally) regarding the expected hospital journey, postoperative care and objective criteria for safe discharge has been demonstrated to reduce the LOS by almost $40 \%$, to 1.5 days. ${ }^{28}$ More proactive interaction with the patient (for example, by telephoning every patient to enquire about postoperative comfort) has also been proposed as an important factor in ensuring patient safety, addressing unexpected symptoms (such as those secondary to hypocalcemia) and avoiding unplanned readmission. ${ }^{11}$ Finally, recent data from the United Kingdom suggest that routine postoperative calcium supplementation after parathyroidectomy can contribute to a reduction in the mean length of stay to $<1$ day. $^{2}$

Limitations of the present study include the small sample size, which is a correlate of the case volume of the unit. The present study is also retrospective and subjected to performance bias. Therefore, it is evident that the number of glands 
biopsied was significantly greater in the pre-SSP cohort. Given that preoperative imaging strategies did not change between cohorts, it is likely that this difference reflects development of surgical experience with time (and therefore case load). Despite this difference, it is important to note that there was no difference in the occurrence of postoperative hypocalcemia.

\section{Conclusions}

Our study has demonstrated that collaborative working, patient education and a pathway incorporating routine postoperative oral calcium supplementation can safely reduce the absolute LOS and variations in LOS after parathyroidectomy. These reductions can be achieved without significant changes in surgical or anesthetic technique. Further reductions in LOS are likely to be possible with additional adjuncts (such as performing surgery under regional blockade rather than general anesthesia), which can be incorporated as more advanced refinements of the clinical pathway to achieve true day case surgery (zero-day stay), and this is currently being addressed within our unit.

\section{Declarations}

The present work has been presented in poster form at the $35^{\text {th }}$ Annual Scientific Meeting of the British Association of Endocrine and Thyroid Surgeons.

\section{Conflict of Interests}

The authors have no conflict of interests to declare.

\section{Acknowledgments}

The authors would like to thank Dr Ryan Haines for his help with administering the institution of the short-stay protocol.

\section{References}

1 Ozbas S, Pain S, Tang T, Wishart GC. Surgical management of primary hyperparathyroidism - results of a national survey. Ann R Coll Surg Engl 2003;85(04):236-241

2 Rajeev P, Sutaria R, Ezzat T, Mihai R, Sadler GP. Changing trends in thyroid and parathyroid surgery over the decade: is same-day discharge feasible in the United Kingdom? World J Surg 2014;38 (11):2825-2830

3 Shindo ML, Rosenthal JM, Lee T. Minimally invasive parathyroidectomy using local anesthesia with intravenous sedation and targeted approaches. Otolaryngol Head Neck Surg 2008;138 (03):381-387

4 Udelsman R, Donovan PI, Sokoll LJ. One hundred consecutive minimally invasive parathyroid explorations. Ann Surg 2000; 232(03):331-339

5 Norman JG, Politz DE. Safety of immediate discharge after parathyroidectomy: a prospective study of 3,000 consecutive patients. Endocr Pract 2007;13(02):105-113

6 Irvin GL III, Sfakianakis G, Yeung L, et al. Ambulatory parathyroidectomy for primary hyperparathyroidism. Arch Surg 1996;131 (10):1074-1078

7 Dulfer RR, van Ginhoven TM, Geilvoet W, de Herder WW, van Eijck $\mathrm{CH}$. Operative Treatment of Primary Hyperparathyroidism in Daycare Surgery. Scand J Surg 2015;104(03):196-199
8 Cohen MS, Finkelstein SE, Brunt LM, et al. Outpatient minimally invasive parathyroidectomy using local/regional anesthesia: a safe and effective operative approach for selected patients. Surgery 2005;138(04):681-687, discussion 687-689

9 Shabtai M, Ben-Haim M, Muntz Y, et al. 140 consecutive cases of minimally invasive, radio-guided parathyroidectomy: lessons learned and long-term results. Surg Endosc 2003;17(05):688-691

10 Mihai R, Palazzo FF, Gleeson FV, Sadler GP. Minimally invasive parathyroidectomy without intraoperative parathyroid hormone monitoring in patients with primary hyperparathyroidism. $\mathrm{Br} \mathrm{J}$ Surg 2007;94(01):42-47

11 Chadwick D, Kinsman R, Walton P, Surgeons ObotBAoEaT. The British Association of Endocrine and Thyroid Surgeons 4th National Audit Report2012.

12 Chadwick D, Kinsman R, Walton P, Surgeons oboTBAoEaT. The British Association of Endocrine and Thyroid Surgeons 5th National Audit Report2017.

13 Vaid S, Pandelidis S. Minimally invasive parathyroidectomy: a community hospital experience. Arch Surg 2011;146(07):876-878

14 Carr ER, Contractor K, Remedios D, Burke M. Can parathyroidectomy for primary hyperparathyroidism be carried out as a day-case procedure? J Laryngol Otol 2006;120(11):939-941

15 Parameswaran R, Allouni K, Varghese P, Misra R, Charlesworth C, Mclaren A. Day Case Parathyroidectomy in a District General Hospital: safe and feasible. The Journal of One Day Surgery. 2010; 20(01):20-22

16 Gurnell EM, Thomas SK, McFarlane I, et al. Focused parathyroid surgery with intraoperative parathyroid hormone measurement as a day-case procedure. Br J Surg 2004;91(01):78-82

17 Carling T, Udelsman R. Focused approach to parathyroidectomy. World J Surg 2008;32(07):1512-1517

18 Palazzo FF, Sadler GP. Minimally invasive parathyroidectomy. BMJ 2004;328(7444):849-850

19 Westerdahl J, Lindblom P, Valdemarsson S, Tibblin S, Bergenfelz A. Risk factors for postoperative hypocalcemia after surgery for primary hyperparathyroidism. Arch Surg 2000;135(02):142-147

20 Bergenfelz A, Lindblom P, Tibblin S, Westerdahl J. Unilateral versus bilateral neck exploration for primary hyperparathyroidism: a prospective randomized controlled trial. Ann Surg 2002;236(05): 543-551

21 Dionigi G, Rovera F, Carrafiello G, Bacuzzi A, Boni L, Dionigi R. New technologies in ambulatory thyroid surgery. Int J Surg 2008;6 (Suppl 1):S22-S25

22 Garas G, Okabayashi K, Ashrafian H, et al. Which hemostatic device in thyroid surgery? A network meta-analysis of surgical technologies. Thyroid 2013;23(09):1138-1150

23 Witteveen JE, van Thiel S, Romijn JA, Hamdy NA. Hungry bone syndrome: still a challenge in the post-operative management of primary hyperparathyroidism: a systematic review of the literature. Eur J Endocrinol 2013;168(03):R45-R53

24 Vasher M, Goodman A, Politz D, Norman J. Postoperative calcium requirements in 6,000 patients undergoing outpatient parathyroidectomy: easily avoiding symptomatic hypocalcemia. J Am Coll Surg 2010;211(01):49-54

25 Kald BA, Heath DI, Lausen I, Mollerup CL. Risk assessment for severe postoperative hypocalcaemia after neck exploration for primary hyperparathyroidism. Scand J Surg 2005;94(03):216-220

26 Crea N, Pata G, Casella C, Cappelli C, Salerni B. Predictive factors for postoperative severe hypocalcaemia after parathyroidectomy for primary hyperparathyroidism. Am Surg 2012;78(03):352-358

27 Willis B, Kim LT, Anthony T, Bergen PC, Nwariaku F, Turnage RH. A clinical pathway for inguinal hernia repair reduces hospital admissions. J Surg Res 2000;88(01):13-17

28 Markey DW, McGowan J, Hanks JB. The effect of clinical pathway implementation on total hospital costs for thyroidectomy and parathyroidectomy patients. Am Surg 2000;66(06):533-538, discussion 538-539 